\title{
Decoding Delay Controlled Completion Time Reduction in Instantly Decodable Network Coding
}

\author{
Ahmed Douik, Student Member, IEEE, Sameh Sorour, Senior Member, IEEE, \\ Tareq Y. Al-Naffouri, Member, IEEE, and Mohamed-Slim Alouini, Fellow, IEEE
}

\begin{abstract}
For several years, the completion time and the decoding delay problems in Instantly Decodable Network Coding (IDNC) were considered separately and were thought to act completely against each other. Recently, some works aimed to balance the effects of these two important IDNC metrics but none of them studied a further optimization of one by controlling the other. This paper investigates the effect of controlling the decoding delay to reduce the completion time below its currently best-known solution in both perfect and imperfect feedback with persistent erasure channels. To solve the problem, the decodingdelay-dependent expressions of the users' and overall completion times are derived in the complete feedback scenario. Although using such expressions to find the optimal overall completion time is NP-hard, the paper proposes two novel heuristics that minimizes the probability of increasing the maximum of these decoding-delay-dependent completion time expressions after each transmission through a layered control of their decoding delays. Afterward, the paper extends the study to the imperfect feedback scenario in which uncertainties at the sender affects its ability to anticipate accurately the decoding delay increase at each user. The paper formulates the problem in such environment and derives the expression of the minimum increase in the completion time. Simulation results show the performance of the proposed solutions and suggest that both heuristics achieves a lower mean completion time as compared to the best-known heuristics for the completion time reduction in perfect and imperfect feedback. The gap in performance becomes more significant as the erasure of the channel increases.
\end{abstract}

Index Terms - Instantly decodable network coding; minimum completion time, decoding delay, perfect/imperfect feedback.

\section{INTRODUCTION}

Network Coding $(N C)$ gained much attention in the past decade after its first introduction in the seminal paper [2]. For example, $\mathrm{NC}$ is shown to be a powerful tool in improving the quality of video streaming to mobile devices, e.g., [3], [4]. An important subclass of network coding, viz., Instantly Decodable Network Coding (IDNC), was an intensive subject of research in multiple areas, e.g., relay-aided networks, video streaming [5]-[8], thanks to its several benefits, such as the use of simple binary XOR to encode/decode packets, no buffer

Copyright (c) 2015 IEEE. Personal use of this material is permitted. However, permission to use this material for any other purposes must be obtained from the IEEE by sending a request to pubs-permissions@ieee.org.

A part of this paper [1] appears in proc. of IEEE Global Telecommunications Conference (GLOBECOM' 2014), Austin, TX, USA, Dec 2014.

Ahmed Douik is with the Department of Electrical Engineering, California Institute of Technology, Pasadena, CA 91125 USA (e-mail: ahmed.douik@caltech.edu).

Sameh Sorour is with the Department of Electrical and Computer Engineering, University of Idaho, Moscow, ID 83844, USA (e-mail: samehsorour@gmail.com).

T. Y. Al-Naffouri and M.-S. Alouini are with the Division of Computer, Electrical and Mathematical Sciences and Engineering, King Abdullah University of Science and Technology, Thuwal 23955-6900, Saudi Arabia (e-mail: \{tareq.alnaffouri,slim.alouini\}@kaust.edu.sa).



Fig. 1. Example illustrating the difference between the completion time and the decoding delays experienced by receivers 1 to 4 in an erasure free scenario. Note that if the order of transmissions is reversed, i.e., packet 3 first then packet $1 \oplus 2$, then receivers 2 and 3 will experience a decoding delay. Hence the completion time is still 2 but the decoding delay becomes 2 . Therefore, for the same completion delay, different decoding delay patterns exists.

requirement, and fast progressive decoding of packets. Such properties are favorable in many applications, e.g., roadside to vehicle safety messages, satellite networks, and IPTV, as compared to the long buffering time needed in other $\mathrm{NC}$ approaches before decoding.

For as long as the research on IDNC has existed, there are two primary metrics that are considered in the literature as measures of its quality, namely the completion time [9] and the decoding delay [10]. The former measures how fast the sender can complete the delivery/recovery of the requested packets whereas the latter measures how far the sender is from being able to serve all the unsatisfied receivers in each and every transmission. Figure 1 illustrates an example of the difference between the completion time and the decoding delays experienced by the different users in a network composed of 4 users and 3 packets.

For quite some time, these two metrics are considered for optimization separately in many works. Though both proved to be NP-hard parameters to minimize, many heuristics have been developed to solve them in many scenarios, e.g., references [11]-[13] study the decoding delay reduction and references [14]-[16] the completion time minimization in a device-todevice communication enabled network. In fact, it can be readily inferred from [9] and [10] that the policies derived so far to optimize one usually degrades the other. It is not until very recently that one work aimed to obtain a policy that can balance between these two metrics and achieve an intermediate performance for both of them [17]. Nonetheless, there does not exist, to the best of our knowledge, any work that aims to explore how these two metrics can be controlled together in order to achieve an even better performance than the currently best-known solutions. For instance, every time an unsatisfied user receives a coded packet that is not targeting it, its decoding delay increases and so does its individual 
completion time. Although this fact is noted for erasure-less transmissions in [17], it is used only to strike a balance in performance between both metrics and not to investigate whether a smart control of such decoding delay effects can further reduce the overall completion time as compared to its current best achievable performance.

Consider a radio access network in which a base-station is required to deliver a frame, composed of several packets, to a set of users. After an initial phase in which the packets are broadcasted one after the other, the base-station efficiently takes advantage of the diversity of the received/lost packets at each user to generate the packets mixes to be transmitted in the recovery phase. The problem of minimizing the completion time in IDNC-based network is the problem of optimally selecting the schedule of packet combinations in order to reduce the completion time under the practical IDNC constraint that mixes are performed using only the binary field $\mathbb{F}_{2}$ and that non-instantly decodable packets are discarded at users. Due to the dynamic nature of the links and the dependence of the optimal schedule of their effects, the above problem is anticausal. The authors in [9] formulate the optimal schedule so as to minimize the completion time as a Shortest Stochastic Path (SSP). Solving such SSP requires computing every possible transmission combination and erasure pattern resulting in a complexity scaling exponentially in the number of users and number of packets. An attractive approach [9] to solving the problem is to take advantage of the geometric property of the SSP formulation to approximate the problem by a more tractable online optimization packet selection algorithm. This paper proposes to improve the approximation by relating the completion time minimization problem to a decoding delay optimization one that can be efficiently solved using on-line algorithms [10], [18].

Previously mentioned works [9], [10], [19] consider a prompt and perfect reception of the feedback. This assumption is not realistic in practice because of the impairments in the feedback channel. The problem of completion time reduction in imperfect feedback is investigated in [20]-[22]. While, the authors in [20], [21] propose blind approaches to solving the problem in lossy feedback and intermittent feedback scenarios, the authors in [22] investigate the problem in Time Division Duplex (TDD) setting with persistent erasure channels. The authors in [23] consider reducing the decoding delay in a lossy intermittent feedback scenario. This paper considers the general feedback imperfections scenario of [23] to study the completion time minimization problem.

The paper's main contribution is to design a new completion time reduction algorithms through decoding delay control in persistent erasure channels in both perfect and imperfect feedback scenarios for IDNC-based networks. In order to solve the problem, the paper first derive a more general expressions of the individual and overall completion times over erasure channels as a function of the users' decoding delays. Since finding the optimal schedule of coded packets to minimize the overall completion time is NP-hard [24], the completion time reduction problem is reformulated as an online optimization problem involving the decoding delay expressions in imperfect feedback derived in [23]. The paper design two greedy heuristics to solve the online optimization problem. The fundamental idea of the algorithms is to reduce the probability of increasing the maximum of the decoding-delay-dependent completion time expressions after each transmission. For the first heuristic, the paper shows that this process can be done by partitioning the IDNC graph into layers with descending order of user completion time criticality before each transmission. The coding combination for this transmission is then designed by going through these descending order layers sequentially and selecting the combination that minimizes the probability of any decoding delay increments within each layer. The selection is done while maintaining the instant decodability constraint of the overall coding combination for the targeted users in the more critical layer(s). For the second heuristic, a binary optimization algorithm with multi-layer objective function is employed to reduce the completion time using the decoding delay formulation. Simulation results show the performance of the proposed solutions and suggest that both heuristics achieves a lower mean completion time as compared to the best-known heuristics for the completion time reduction in perfect and imperfect feedback. The gap in performance becomes more significant as the erasure of the channel increases.

The rest of this paper is organized as follows: In Section II, the system and the channel model are presented. The problem formulation using a decoding delay approach is presented in Section III. Analytic formulation of the optimal solution of the approximated problem at each transmission is provided in Section IV. In Section V, algorithms to solve the former problem are presented. Section VI shows the extension of the study to the imperfect feedback scenario. Before concluding in Section VIII, simulations results are illustrated in Section VII.

\section{System \& ChanNEL Model}

The first part of the paper studies the completion time reduction problem in perfect feedback scenario. Therefore, this sections presents the system and channel model of an IDNCbased network with persistent erasure channels and perfect feedback reception. The system model and feedback protocol in imperfect feedback are shown in Section VI.

\section{A. System Model and Parameters}

Consider a radio access network in which a base-station is required to deliver a frame (denoted by $\mathcal{N}$ ) of $N$ source packets to a set (denoted by $\mathcal{M}$ ) of $M$ users. Each user is interested in receiving all the $N$ packets of $\mathcal{N}$. In an initial phase, the sender transmits sequentially the $N$ packets of the frame uncoded. Each user listens to all transmitted packets and feeds back to the sender an acknowledgment for each successfully received one.

After the first transfer, the recovery period starts. In this phase, the BS uses binary XOR to encode the source packets to be sent. The packet combination is chosen using the diversity of lost/received by each user and the expected erasure patterns of the links. The packets, at each time instant, can be in one of the following set:

- The Has set (denoted by $\mathcal{H}_{i}$ ) is defined as the set of packets successfully received by user $i$. 
- The Wants set (denoted by $\mathcal{W}_{i}$ ) is defined as the set of packets that are lost by user $i$.

The base-station stores these information about lost/received packets in a matrix form $\mathbf{F}=\left[f_{i j}\right], \forall i \in \mathcal{M}, \forall j \in \mathcal{N}$, called herein the feedback matrix (FM), such that:

$$
f_{i j}= \begin{cases}0 & \text { if } j \in \mathcal{H}_{i} \\ 1 & \text { if } j \in \mathcal{W}_{i} .\end{cases}
$$

Remark 1. The variables defined in this section are a function of the transmission time $t$, e.g., the Has set should be written as $\mathcal{H}_{i}(t)$. However, for notation convenience, the time index will be dropped unless it is required. The sets $\mathcal{H}_{i}(0)$, and $\mathcal{W}_{i}(0)$ refer to the sets at the beginning of the recovery phase, i.e., the sets obtained from the initial transmissions.

After the initial phase, the recovery phase begins. In this phase, the sender exploits the diversity in received packets at the different users to transmit network coded combinations of the source packets. After each transmission, targeted users, i.e., users to whom the BS indented the packet combination when encoding it, acknowledge the reception/loss of the packet. This process is repeated until all users complete the reception of all frame packets. In this phase, for each user $i$, the transmitted coded packets can be one of the following three options:

- Non-innovative: The packet does not bring new useful information. In other words, all the source packets encoded in it were successfully received and decoded previously.

- Instantly Decodable: The packets contains at most one packet the user do not have so far. In other words, it contains only one packet from $\mathcal{W}_{i}$.

- Non-Instantly Decodable: The packets contains more than one source packet the user does not have so far. In other words, it contains at least two packets from $\mathcal{W}_{i}$.

Given a schedule $S$ of coded packets transmitted by the sender, the individual completion time, overall completion time and the decoding delay [10], [17], [19], [21], [25] are defined as follows:

Definition 1. The individual completion time $\mathcal{C}_{i}(S)$ of user $i$ is the number of recovery transmissions required until this user obtained all its requested packets.

Definition 2. The overall completion time $\mathcal{C}(S)$ of a frame is the number of recovery transmissions required until all users obtain all their requested packets. It holds by definition that $\mathcal{C}(S)=\max _{i \in \mathcal{M}} \mathcal{C}_{i}(S)$

Definition 3. At any transmission, a user $i$, with non-empty Wants set, experiences a one unit increase of decoding delay if it successfully receives a packet that is either non-innovative or non-instantly decodable. Consequently, the decoding delay $D_{i}(S)$ experienced by user $i$ given a schedule $S$ is the number of received coded packets by $i$ before its individual completion, which are non-innovative or non-instantly decodable.

Note that the decoding delay accounts only for delays due to coding decisions. Hence, the channel erasure instances are excluded from its definition. The rest of this paper shows that an effective control of such decoding delay helps reducing the completion time.

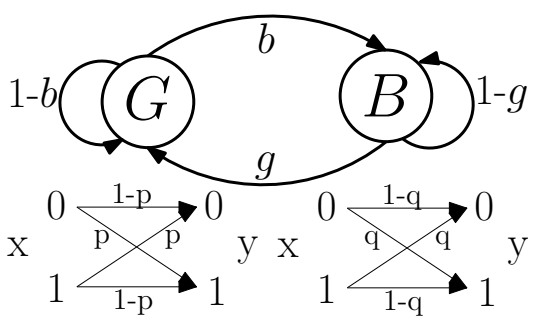

Fig. 2. The two state Gilbert-Elliott channel. The Good state $(G)$ results in a successful transmission with probability $1-p$ whereas the Bad state $(B)$ results in a successful one with probability $1-q, p \leq q$.

\section{B. Forward Channel Model}

To capture the persistent nature of the forward channel, the paper adopts, as in [22], [23], the Gilbert-Elliott channel (GEC) model. The Good and Bad states are designed by $G$ and $B$, respectively, as shown in Figure 2. In the original version of the Gilbert-Elliott channel [26], the Good state is assumed to be error free whereas the Bad one is always in error, i.e., $p=0$ and $q=1$ in Figure 2. This zero error probability in the Good state allows the computation of the capacity in closedform [27]. To study multiple fading scenarios, the model is extended in [28] to incorporate a non-zero error probability in the Good state. Unlike [22], [23], this paper considers the general formulation of the GEC.

Let $C_{i}$ denote the state of the forward channels. For each user $i$, the transition probability, from time $t-1$ to $t$, are given below:

$$
\begin{aligned}
& \mathbb{P}\left(C_{i}(t)=G \mid C_{i}(t-1)=B\right)=g_{i} \\
& \mathbb{P}\left(C_{i}(t)=B \mid C_{i}(t-1)=G\right)=b_{i} .
\end{aligned}
$$

The probabilities for a packet to be erased at user $i$ in the Good and the Bad states are, respectively, $p_{i}$ and $q_{i}$. Since the Markov chain is time-homogeneous, i.e., the process can be described by a single, time-independent matrix, the probabilities to be in the Good or the Bad states (steady-state probabilities) can be expressed as follows:

$$
\mathcal{P}_{G_{i}}=\mathbb{P}\left(C_{i}=G\right)=\frac{g_{i}}{g_{i}+b_{i}}=1-\mathcal{P}_{B_{i}} .
$$

Let $\mu_{i} \triangleq 1-g_{i}-b_{i}$ be the memory factor indicating the correlation between the states. A high value of this factor means that the channel is likely to stay in the same state during the following transmissions. In contrast, a small value indicates that the state of the channel changes in an independent manner. Similarly, let $\mu$ be the average memory of the forward link, i.e., the mean of $\mu_{i}$ 's. The paper assumes that no correlation exists between the channels of different users and that the transition probabilities are entirely known at the BS.

\section{Problem Formulation USing DECODING-DELAY-DEPENDENT EXPRESSIONS}

In this section, the completion time reduction problem is formulated using a decoding delay approach. Let $\mathcal{S}$ be the set of all possible transmission schedules of coded packets. The completion time minimization problem is the one of finding the optimal schedule so as to minimize the completion time. In 
other words, the optimal schedule may be written as follows:

$$
S^{*}=\arg \min _{S \in \mathcal{S}}(\mathcal{C}(\mathcal{S}))=\arg \min _{S \in \mathcal{S}}\left\{\max _{i \in \mathcal{M}} \mathcal{C}_{i}(S)\right\} .
$$

As discussed earlier, the optimization problem (4) is anticausal. This paper proposes approximating its solution by reformulation the problem as an online optimization involving an anticipated version of the completion time. In order to derive the expression of the anticipated completion time, this section first expresses the completion time using a decoding delay expression.

Let $d_{i}(\kappa(t))$ be the decoding delay increase for user $i$, at time $t$, after the transmission $\kappa(t)$. Define $D_{i}(t)$ as the total decoding delay experienced by user $i$ until the transmission at time $t$, i.e., $D_{i}(t)=\sum_{n=1}^{t} d_{i}(\kappa(n))$. The following theorem introduces a decoding-delay-dependent expression for the individual completion time of user $i$ and the overall completion time, given the transmission of schedule $S$ from the sender over erasure channels.

Theorem 1. For a relatively large number of packets $N$, and a schedule $S$ of transmitted packets, the individual completion time for user $i$ can be approximated by:

$$
\mathcal{C}_{i}(S) \approx \frac{\left|\mathcal{W}_{i}(0)\right|+D_{i}(S)-\alpha_{i}}{1-\alpha_{i}}
$$

where $\alpha_{i}$ is the average erasure probability over the persistent channel defined as:

$$
\alpha_{i}=\frac{g_{i} p_{i}+q_{i} b_{i}}{g_{i}+b_{i}}
$$

Proof: The proof can be found in Appendix A.

In the rest of the paper, the approximation is used with equality as it indeed holds for large $N$. Substituting the decoding-delay-dependent expression of the completion time (5) in the optimization problem (4) yields the following expression of the optimal schedule:

$$
S^{*}=\arg \min _{S \in \mathcal{S}}\left\{\max _{i \in \mathcal{M}}\left\{\frac{\left|\mathcal{W}_{i}(0)\right|+D_{i}(S)-\alpha_{i}}{1-\alpha_{i}}\right\}\right\},
$$

Clearly, finding the optimal schedule through the above optimization formulation is very difficult. This is due to the dynamic nature of the erasures and the dependence of the optimal schedule of their effects, which makes the above equation anti-causal, i.e., the current result depends on input from the future. Moreover, it is known from the literature that optimizing the completion time over the whole recovery phase is intractable [21] even for the erasure-less scenario [17]. On the other hand, this formulation shows that the only terms affected by the schedule in the individual and overall completion time expressions are the decoding delay terms of the different users. Consequently, controlling such decoding delays in a smart way throughout the selection of the coded packet schedule can indeed affect the reduction of the completion time significantly. In next section, this fact is taken into consideration in the design of new heuristic algorithms for effective completion time reduction.

\section{Completion Time Reduction}

In this section, the optimization problem (7) is approximated by a more tractable problem using an anticipated version of the completion time. The section further presents the IDNC graph as a tool to represent the feasible packet combinations. Afterward, the approximate problem is shown to be equivalent to a maximum weight clique problem in the IDNC graph that can be efficiently solved using existing literature, e.g., [29][31].

\section{A. Critical Criterion}

From the optimization problem in (7), it is clear that the optimal schedule is the one that achieves the minimum overall growth in all the individual completion time. Since finding such schedule for the entire recovery phase, prior to its start, is intractable, this paper proposes to design heuristic algorithms that minimize the probability of increase of the maximum of such expressions over all users compared to their state before the transmission. To formally express this criterion, this section first introduce the anticipated completion time as follows:

$$
\mathcal{C}_{i}(t)=\frac{\left|\mathcal{W}_{i}(0)\right|+D_{i}(t)-\alpha_{i}}{1-\alpha_{i}}
$$

In other words, $C_{i}(t)$ is the anticipated individual completion time of user $i$ if it experiences no further decoding delay increments starting from time $t$. Thus, the philosophy of the proposed heuristic algorithms is to transmit the coded packet $\kappa(t)$ so that to minimize the following probability:

$$
\min \left\{\mathbb{P}\left(\max _{i \in \mathcal{M}}\left\{\mathcal{C}_{i}(t)\right\}>\max _{i \in \mathcal{M}}\left\{\mathcal{C}_{i}(t-1)\right\}\right)\right\} .
$$

The criterion (9) is called, herein, the "critical criterion" as it influences directly the overall completion time. Let $j$ be the user with the maximum anticipated completion time at transmission $t-1$, i.e., $j=\arg \max _{i \in \mathcal{M}} \mathcal{C}_{i}(t-1)$. Let $\mathcal{P}(t)$ be the set of users that can potentially increase the anticipated completion time at time $t$ compared to its maximal value $\mathcal{C}_{j}(t-1)$ if they are not targeted by $\kappa(t)$. Since only the decoding delay terms affects such anticipated completion time, the set $\mathcal{P}(t)$ can be mathematically defined as follows:

$$
\mathcal{P}(t)=\left\{\begin{array}{l|l}
i \in \mathcal{M} & \mathcal{C}_{i}(t-1)+\frac{1}{1-\alpha_{i}}>\mathcal{C}_{j}(t-1)
\end{array}\right\},
$$

In the rest of this paper, the set $\mathcal{P}(t)$ is referred to as the "highly critical set" as it contains the users that can satisfy the critical criterion defined above.

\section{B. Instantly Decodable Network Coding Graph}

The authors in [10] introduce the IDNC graph as a tool to determine both the possible packet combination and the users that can instantly decode such combination. To construct the IDNC graph $\mathcal{G}(\mathcal{V}, \mathcal{E})$, a vertex $v_{i j} \in \mathcal{V}$ is generated for each packet $j \in \mathcal{W}_{i}, \forall i \in \mathcal{M}$. Two distinct vertices $v_{i j}$ and $v_{k l}$ are connected if one of the two following conditions holds:

- $j=l \Rightarrow$ Packet $j$ is needed by both users $i$ and $k$.

- $j \in \mathcal{H}_{k}$ and $l \in \mathcal{H}_{i} \Rightarrow$ The packet combination $j \oplus l$ is instantly decodable for both users $i$ and $k$.

Given the graph constructed as above and according to the analysis done in [24], the set of all packet feasible combinations in IDNC-based networks is represented by all maximal cliques in $\mathcal{G}$. For a selected maximal clique, the packet combination is generated by allying binary XOR to 
the packets identified by the clique and the targeted users are those determined by the vertices of the clique.

\section{Optimization Problem}

In this subsection, the optimization problem (9) is reformulated as a maximum weight clique problem in the IDNC graph. In order to express the weight of the vertices in the IDNC graph, the section first presents the probability to lose a transmission at a given user. Given that a communication can be successful in both states of the channel (but with different expectations), the sender cannot determine surely the state of the user's channel. Moreover, since only targeted users acknowledge the reception of their intended packets, the problem becomes more severe for non-targeted users.

Let $t_{i}^{(0)}$ the latest time user $i$ is targeted and let $j$ be the intended packet for user $i$ in that transmission. The following proposition expresses the probability for the transmission at time $t$ to be erased at user $i$ given the available information, i.e., the reception status of the latest transmission.

Proposition 1. The probabilities $e_{i}(t)$ of loosing a transmission from user $i$ at time $t>t_{i}^{(0)}$ can be expressed as:

$$
e_{i}(t)= \begin{cases}\frac{p_{i} g_{i} \eta_{i}+q_{i} b_{i} \eta_{i}^{\prime}}{p_{i} g_{i}+q_{i} b_{i}} & \text { if } f_{i j}=1 \\ \frac{\left(1-p_{i}\right) g_{i} \eta_{i}+\left(1-q_{i}\right) b_{i} \eta_{i}^{\prime}}{\left(1-p_{i}\right) g_{i}+\left(1-q_{i}\right) b_{i}} & \text { if } f_{i j}=0 .\end{cases}
$$

where the quantities $\eta_{i}$ and $\eta_{i}^{\prime}$ are defined as follows:

$$
\begin{gathered}
\eta_{i}=p_{i}+\left(q_{i}-p_{i}\right) b_{i} \sum_{k=0}^{t-t_{i}^{(0)}-1} \mu_{i}^{k} \\
\eta_{i}^{\prime}=q_{i}+\left(p_{i}-q_{i}\right) g_{i} \sum_{k=0}^{t-t_{i}^{(0)}-1} \mu_{i}^{k} .
\end{gathered}
$$

Proof: The proof can be found in Appendix B.

For zero error probability channels, e.g., the one studied in [23], the expression $\mathrm{f}$ the erasure probability can be obtained by setting $p_{i}=0$ and $q_{i}=1$ :

$$
e_{i}(t)= \begin{cases}1-g_{i} \sum_{k=0}^{t-t_{i}^{(0)}-1} \mu_{i}^{k} & \text { if } f_{i j}=1 \\ b_{i} \sum_{k=0}^{t-t_{i}^{(0)}-1} \mu_{i}^{k} & \text { if } f_{i j}=0,\end{cases}
$$

which is in agreement with the expression derived in [23].

Let $\tau(\kappa)$ be the set of users that are targeted by the transmission $\kappa$. The following theorem reformulated the optimization problem (9) as a maximum weight clique problem in the IDNC graph.

Theorem 2. The critical criterion in (9) can be achieved by selecting $\kappa^{*}$ according to the following optimization problem:

$$
\kappa^{*}=\underset{\kappa \in \mathcal{G}}{\operatorname{argmax}}\left\{\sum_{i \in(\mathcal{P}(t) \cap \tau(\kappa))} \log \left(\frac{1}{e_{i}(t)}\right)\right\} .
$$

In other words, the transmission $\kappa$ than satisfy the critical (9) criterion is the maximum weight clique in the IDNC graph in which the weight of each vertex $v_{i j}$ is:

$$
w_{i j}^{*}= \begin{cases}-\log \left(e_{i}(t)\right) & \text { if } i \in \mathcal{P}(t) \\ 0 & \text { otherwise. }\end{cases}
$$

Proof: The proof can be found in Appendix C.

\section{Proposed Heuristics Algorithms}

As shown in previous section, the optimal solution to the completion time reduction problem (13) involves a maximum weight clique search over the IDNC graph. Such problem being NP-hard, in this section, the paper proposes two heuristics to solve the optimization problem. The first heuristic approximates the maximum weight clique problem. To further improve the performance, the heuristic suggests adding vertices not included in the highly critical set are considered so as to ameliorate the approximation in (9). The second heuristic relies on a binary search over the feasible space using a popular method namely the particle swarm optimization. In order to improve the performance of such method, the paper proposes a particular initialization and uses a multi-layer objective function similar to the one employed for the first heuristic.

\section{A. Maximum Weight Clique Solution}

Despite the importance of the satisfaction of the critical criterion in order to minimize the probability of the increase in the maximum individual completion time, it may not fully exploit the power of IDNC. In other words, once a clique is chosen according to (13) among the vertices representing users in the highly critical set $\mathcal{P}(t)$, there may exist vertices belonging to other users that can form an even bigger clique. Thus, adding these vertices to the clique and serving this user will benefit them without affecting the IDNC constraint for the users belonging to $\mathcal{P}(t)$. A natural prioritization among these vertices is to choose the ones that are the most likely to be in the highly critical set in the following transmissions.

To schedule such vertices and their users, let $\mathcal{G}_{1}, \mathcal{G}_{2}, \ldots \mathcal{G}_{h}$ (with $h \in \mathbb{N}$ ) be the sets of vertices of $\mathcal{G}(t)$, such that $v_{i k} \in \mathcal{G}_{n}$ if the following conditions are true:

$$
\begin{aligned}
& \text { - } \mathcal{C}_{i}(t-1)+\frac{n}{1-\alpha_{i}}>\mathcal{C}_{j}(t-1) . \\
& \text { - } \mathcal{C}_{i}(t-1)+\frac{n-1}{1-\alpha_{i}} \leq \mathcal{C}_{j}(t-1) . \\
& \text { where } j=\underset{i \in \mathcal{M}}{\operatorname{argmax}}\left\{\mathcal{C}_{i}(t-1)\right\} \text {. Consequently, the IDNC }
\end{aligned}
$$
graph at time $t$ is partitioned into $h$ layers with descending order of criticality. By examining the above condition, the vertices of the users of $\mathcal{P}(t)$ are all in layer $\mathcal{G}_{1}$. Moreover, the $n$-th layer of the graph includes the vertices of the users who may eventually increase $\max _{i \in \mathcal{M}}\left\{\mathcal{C}_{i}(t+n)\right\}$ if they experience $n$ decoding delay increments in the subsequent $n$ transmissions. Consequently, a user with vertices belonging to $\mathcal{G}_{i}$ is more critical than another with vertices belonging to $\mathcal{G}_{j}$, $j>i$, as the former has a higher chance to increase the overall completion time.

In order to guarantee the satisfaction of the critical criterion, our proposed algorithm first finds the maximum weight clique $\kappa^{*}$ in layer $\mathcal{G}_{1}$ as mandated by Theorem 2. Afterward, the graph $\mathcal{G}_{2}\left(\kappa^{*}\right)$ is constructed including vertices in $\mathcal{G}_{2}$ that are adjacent to all vertices in $\kappa^{*}$. After assigning the same weights defined in (14), i.e., $-\log \left(e_{i}(t)\right)$, the maximal weight clique 
in $\mathcal{G}_{2}\left(\kappa^{*}\right)$ is found and added to $\kappa^{*}$. This process is repeated for each layer $\mathcal{G}_{i}, i \leq h$ of the graph to find the selected maximal weight clique $\kappa^{*} \in \mathcal{G}(t)$ to be transmitted at time $t$.

Since finding the maximum weight clique in the G-IDNC graph is NP-hard [24], a simple vertex search approach, similar to the one in [9], is considered. The approach relies on modifying the weights defined in (14) so as to account for the connectivity of the vertices. Let $w_{i j}$ be the adjusted weights, which can be expressed as:

$$
w_{i j}=w_{i j}^{*} \sum_{v_{k l} \in \nu\left(v_{i j}\right)} w_{k l}^{*},
$$

where $\nu\left(v_{i j}\right)$ is the set of adjacent vertices of $v_{i j}$ within its layer.

\section{B. Binary Particle Swarm Optimization Solution}

Particle Swarm Optimization (PSO) is a population search algorithm based on the simulation of the social behavior of animals, introduced in [32] by Eberhart and Kennedy. A couple of vectors is associated with each of the $L$ individuals in the multidimensional space namely the position vector and the velocity vector. While the position vector indicates the current position of the particle, the velocity vector determines in which direction the position vector should evolve. The process of updating the position and velocity vectors is repeated $T$ times.

Khanesar et al. [33] propose a novel binary PSO (BPSO) based on a new definition of the velocity vector. The paper considers that algorithm whose steps can be found in [33]. This section suggests defining the objective function $\phi$ so as to both minimize the critical criterion (13) and to improve the approximation in (9) using a same method like the one in the previous heuristic.

In order to define the objective function, first introduce the sigmoid function is defined as follows:

$$
\operatorname{sig}(x)=\frac{1}{1+e^{-x}}
$$

Following the solution proposed in the previous heuristic, the objective function should consider including users in the highly critical layer first. Once such users are added, the heuristic adds users that are not in the critical layer under the condition that their inclusion to not disturb the instantaneous decodability of the targeted users in the critical layer. Such integration is performed under layer prioritization, i.e., the inclusion of a user in layer $\mathcal{P}_{m}$ should not disturb the instant dependability of users in layer $\mathcal{P}_{n}, n>m$. To reproduce this concept of prioritization using a single real function, this section proposes the following objective function:

$$
\begin{aligned}
\phi(\kappa) & =\sum_{i \in \tau(\kappa)} \operatorname{sig}\left(-\log \left(e_{i}(t)\right)\right)+M *(h-P(i)) \\
& =\sum_{i \in \tau(\kappa)} \frac{1}{1+e_{i}(t)}+M *(h-P(i)) .
\end{aligned}
$$

where $h$ is the total number of layers and $P(i)$ is the index of the layer of user $i$. The sigmoid function being an increasing function over $[0,1]$, the utility of each user is shifted according to its layer number. Given that at maximum $M$ users can be simultaneously on the same layer, all these layers are nonoverlapping, and each element of one layer yields a better objective than the sum of all user in a lower layer. Therefore, the objective function $\phi$ respects the layer prioritization. The following lemma suggests a particular number of search particle $L$ and an appropriate initialization in order to guarantee the convergence of the overall system.

Lemma 1. For a number of particles equal to the number of packets, i.e., $L=N$, and an identity initialisation matrix, the overall system is convergent for an arbitrary number of iterations $T$.

Proof: The proof can be found in Appendix D.

\section{Extension To The Imperfect Feedback SCENARIO}

This section solves the completion time reduction problem in imperfect feedback scenario by extending the results of previous sections. The paper suggests employing a decoding delay approach as for the perfect feedback situation and minimizing the probability of increase of the maximum anticipated decoding delay. To solve the problem, this section first presents the system model and the feedback protocol. The optimal packet combination that minimizes the increase in the maximum anticipated completion time is formulated using the decoding-delay-dependent expressions. Finally, the paper suggests extending the heuristics proposed, in the previous section, to solve the problem with moderate complexity.

\section{A. System Model \& Feedback Protocol}

In imperfect feedback situation, uncertainties at the sender affect its ability to determine the instantly decodable combination accurately. Such uncertainties are incorporated into the system by attributing, at the end of the initial phase, three sets of packets for each user $i$ as follows:

- The Has set (denoted by $\mathcal{H}_{i}$ ) is defined as the set of packets successfully received by user $i$.

- The Wants set (denoted by $\mathcal{W}_{i}$ ) is defined as the set of packets that are not successfully received by user $i$.

- The Uncertain set (denoted by $\mathcal{U}_{i}$ )is defined as the set of packets whose state is uncertain at the BS, i.e., $\mathcal{U}_{i} \subseteq \mathcal{W}_{i}$.

Following the same general model as the forward, the feedback (backward) channel is modeled by non-zero error probability Gilbert-Elliott channel. The channel parameters are similar to the ones displayed in Figure 2. To differentiate between the forward and backward channel, the parameters are written with a tilde " $\cdots$, e.g, the transiting probability are $\tilde{g}_{i}$ and $\tilde{b}_{i}$ for user $i$. Let $\psi_{i} \triangleq 1-\tilde{g}_{i}-\tilde{b}_{i}$ be the memory of the channel of user $i$ and $\psi$ the average memory, defined in the manner as $\mu_{i}$ and $\mu$.

The paper considers the most general feedback model in which the acknowledgement is not only received after a given number of transmission, but it is also subject to erasure. Let the time is divided into frames of same length $T_{f}$ time slots. Each of these frames is composed of a downlink sub-frame and an uplink sub-frame. The BS consecutively transmits the packet combinations during the downlink sub-frame without any excepted acknowledgement. After the downlink sub-frame, users that received and managed to decode a packet combination 
during the previous downlink sub-frame acknowledges its reception by sending a feedback during the uplink sub-frame. As for the previous sections, a feedback from a targeted user contains an acknowledgement of all previously received packets.

In order for the BS to be able to estimate accurately the state of the forward/backward channels for each user, this paper proposes the same restriction on the generated combination as in [23]. The limit consists of attempting at least one packet once for each user from the last time a feedback is heard from that user. Such constraint becomes unnecessary for users wanting a single source packet.

\section{B. Problem Formulation}

This subsection formulates the completion time reduction problem. The decoding-delay-dependent completion time expression derived in Theorem 1 being independent of the feedback scenario, the paper proposes minimizing the anticipated completion time through decoding delay control in the imperfect feedback situation. In other words, this section suggests reducing the probability of increase in the completion time from its maximal value value so far, i.e., finding the optimal packet combination that satisfy the critical criterion defined in (9).

To express the decoding delay and hence the critical criterion, this subsection first introduce additional system variables. Let $t_{i}^{(0)}$ be the most recent time instant in which user $i$ was targeted only once by a packet $j$ and let $t_{i}^{(*)}$ be time instant in which an acknowledgement is heard from that user. While the probability $e_{i}(t)$ for a transmission to be erased at time $t$ is given by Proposition 1, the following corollary computes the probability $f_{i}(t)$ for a feedback to be erased.

Corollary 1. The probabilities $f_{i}(t)$ of loosing a transmission from user $i$ at time $t>t_{i}^{(*)}$ can be expressed as:

$$
f_{i}(t)=\frac{\left(1-\tilde{p}_{i}\right) \tilde{g}_{i} \tilde{\eta}_{i}+\left(1-\tilde{q}_{i}\right) \tilde{b}_{i} \tilde{\eta}_{i}^{\prime}}{\left(1-\tilde{p}_{i}\right) \tilde{g}_{i}+\left(1-\tilde{q}_{i}\right) \tilde{b}_{i}},
$$

where the quantities $\tilde{\eta}$ and $\tilde{\eta}^{\prime}$ are defined in the same manner as in (11) using the backward channel parameters.

Proof: Since the feedback is successfully transmitted at time $t^{(*)}$, then the realisation of the backward channel is known, i.e., $\mathcal{X}\left(t^{(*)}\right)=0$. The rest of the proof is omitted as it mirrors the steps used in proving Proposition 1.

Since the uncertainties at the sender affects its ability to determine the instant decodability of packet combinations at the different users, such a sender is not longer sure about whether a packet combination is innovative for the targeted user. Moreover, it is no longer sure whether users completed receiving their missing packet or not. To account for such uncertainties, this section introduces the following probabilities:

- The innovative probability $p_{i, n}(j)$ defined as the probability that packet $j$ is innovative for user $i$.

- The finish probability $p_{i, f}$ defined as probability that user $i$ successfully received all packets but $\mathcal{W}_{i} \neq \varnothing$.

The expressions of the innovative and the finish probability as a function of the transmission erasure probability $e_{i}$ and the feedback erasure probability $f_{i}$ can be found in [23]. Let $\kappa_{i}$ be the packet intended for user $i$ in the transmission $\kappa$. The following theorem formulates the optimal packet combination to be transmitted, in imperfect feedback situation, at time $t$, so as to minimize the critical criterion defined in (9).

Theorem 3. The critical criterion in (9) for imperfect feedback scenario can be achieved by selecting $\kappa^{*}$ according to the following optimization problem:

$$
\max \left\{\sum_{i \in(\mathcal{P}(t) \cap \tau(\kappa))} \log \left(1+\frac{p_{i, n}\left(\kappa_{i}\right)}{\frac{e_{i}(t)}{1-e_{i}(t)}+p_{i, f}}\right)\right\} .
$$

In other words, the transmission $\kappa$ than satisfy the critical (9) criterion in imperfect feedback situation is the maximum weight clique in the IDNC graph in which the weight of each vertex $v_{i j}$ is:

$$
w_{i j}^{*}= \begin{cases}\log \left(1+\frac{p_{i, n}(j)}{\frac{e_{i}(t)}{1-e_{i}(t)}+p_{i, f}}\right) & \text { if } i \in \mathcal{P}(t) \\ 0 & \text { otherwise. }\end{cases}
$$

Proof: The proof can be found in Appendix E.

\section{Proposed Algorithm}

This subsection proposes to extend the heuristics developed in the previous section to the imperfect feedback scenario. Similar to the previous section, both heuristics reduce the complexity of the maximum weight clique search and improve the completion time approximation proposed in (9).

1) Maximum Weight Clique Solution: In the context of decoding delay reduction, the authors in [34] show that the IDNC graph fails to produce satisfactory results as it considers only packet that are surely combinable. To address the problem, they introduced the lossy IDNC graph (LG-IDNC) that represents a bigger number of possible combinations including all the combinations in the IDNC graph. The fundamental idea in the construction of such graph is to combine each vertex as long as the decoding delay represented by the combination is lower than the individual delays of the packets. Given that our completion time reduction problem relies on a decoding delay minimization, such graph formulation can be easily extended to the scenario under investigation.

To construct the LG-IDNC graph, first introduce the expected decoding delay increase $d_{i j, k l}(j \oplus l)$ for two distinct arbitrary users $i$ and $k$ after sending the packet combination $j \oplus l$ :

$$
\begin{aligned}
& d_{i j, k l}(j \oplus l)= \\
& \left(1-e_{i}\right)\left(p_{n, i}(j) p_{n, i}(l)+\left(1-p_{n, i}(j)\left(1-p_{n, i}(l)\right)\right) \bar{p}_{f, i}+\right. \\
& \left(1-e_{k}\right)\left(p_{n, k}(j) p_{n, k}(l)+\left(1-p_{n, k}(j)\left(1-p_{n, k}(l)\right)\right) \bar{p}_{f, k},\right.
\end{aligned}
$$

where $\bar{p}_{f, i}=1-p_{f, i}$.

The expected decoding delay increase $d_{i j, k l}(j)$ for these users after sending packet $j$, is obtained by replacing $l$ by 0 in (21) and considering $p_{n, i}(0)=0, \forall i \in \mathcal{M}$. The LGIDNC graph $\mathcal{G}(\mathcal{V}, \mathcal{E})$, is constructed by generating a vertex 
$v_{i j}, \forall i \in \mathcal{M}, \forall j \in \mathcal{W}_{i}$ then connecting two vertices $v_{i j}$ and $v_{k l}$ if one of the following conditions is true:

- $\mathrm{C} 1: j=l \Rightarrow$ Packet $j$ is needed by users $i$ and $k$.

- $\mathrm{C} 2: d_{i j, k l}(j \oplus l) \leq \min \left(d_{i j, k l}(j), d_{i j, k l}(l)\right) \Rightarrow$ The packet combination $j \oplus l$ guarantees a lower decoding delay to the users $i$ and $k$ than packets $j$ and $l$ individually.

Unlike condition $\mathrm{C} 1$ that does not require packet combination, $\mathrm{C} 2$ involves the combination of packet $j$ and $l$. After the construction of the LG-IDNC graph, the heuristic follows the same step as the multi-layer algorithm developed in Section V considering the new weights defined in (20).

2) BPSO Algorithm: The same line of thinking used in Section V applies in the case of the imperfect feedback scenario. Using a method similar to the one in Section V, it can easily be shown that the objective function $\phi$ that reflects the uncertainties in the system, i.e., uses the weights (20), and respects the layer prioritization is the following:

$$
\phi(\kappa)=\sum_{i \in \tau(\kappa)} \frac{\frac{e_{i}(t)}{1-e_{i}(t)}+p_{i, f}+p_{i, n}}{\frac{2 e_{i}(t)}{1-e_{i}(t)}+2 p_{i, f}+p_{i, n}}+M *(h-P(i)) .
$$

where $h$ is the total number of layers, $P(i)$ is the index of the layer of user $i$ and $\tilde{\phi}_{i}$ defined as follows:

\section{Blind Graph Policies Solution}

This subsection presents three partially blind algorithms first proposed in [20] in the context of reducing the completion time in the lossy feedback scenario. To be fair in comparison, the section extends such approach to the feedback situation under investigation. The fundamental concept of these algorithms is to estimate the uncertainties with a predefined policy, update the graph accordingly and finally perform packet selection using the algorithm proposed in [18].

1) Pessimist Graph Update: In this approach, packets that are not fed back are considered erased rather than assuming that their feedback is erased. Reconsidering these packets in the following transmissions gives them a greater chance to be reattempted rapidly. Since no acknowledgement is expected to be heard during the downlink subframe, packets attempted meanwhile are systematically not reconsidered in the following transmissions. If a feedback is heard in the uplink subframe, the state of the user is updated. Otherwise, all the uncertain packet of that user are reconsidered.

In the pessimist graph update approach, uncertain vertices are removed from the graph during the downlink subframe and reconsidered in the uplink subframe if no acknowledgement is heard from the concerned user.

2) Optimist Graph Update: In this approach, packets that are not fed back are considered received, and their corresponding feedback erased. Not reconsidering these packets in the following transmissions gives a greater chance to nonattempted packets to be transmitted. Since no feedback can be heard from a user having all its packets in an uncertain state, unless this user is targeted. Therefore, users with full uncertain Wants set are reconsidered after the uplink subframe.

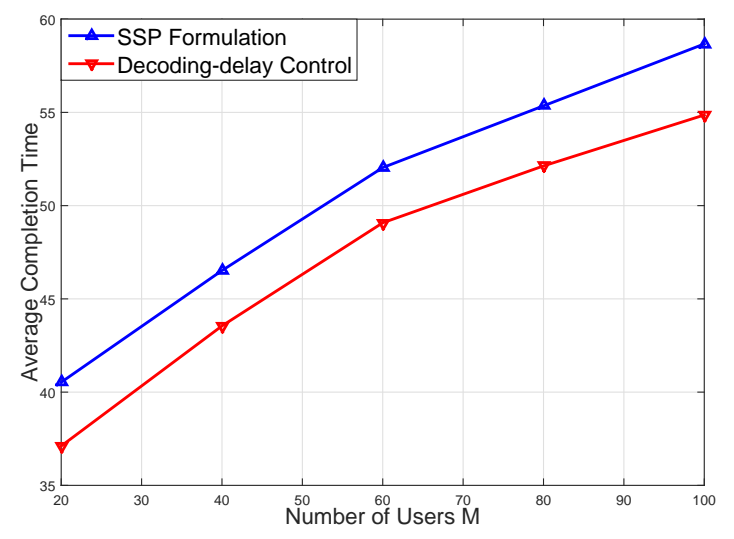

Fig. 3. Completion time versus the number of users $M$ for a network composed of $N=30$ packets and an average erasure probability $P=0.25$ for memoryless channels with perfect feedback.

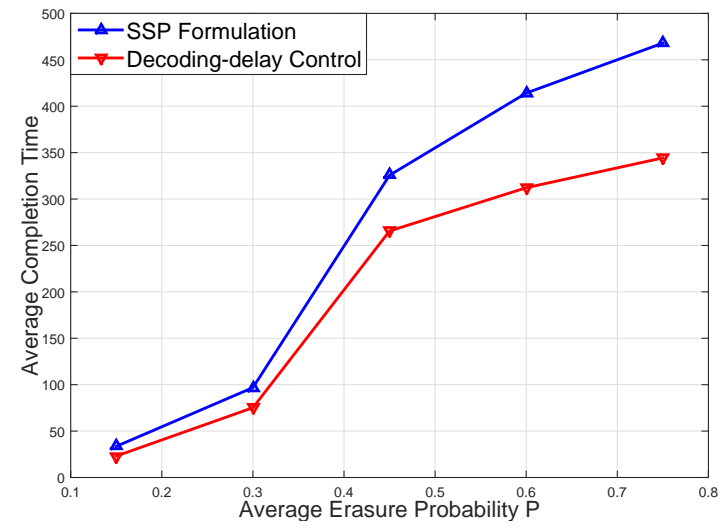

Fig. 4. Completion time versus the average erasure probability $P$ for a network composed of $M=60$ users and $N=30$ packets for memoryless channels with perfect feedback.

In the optimist graph update approach, uncertain vertices are removed from the graph and reconsidered in the uplink subframe if the user have full uncertain Wants set.

3) Realistic Graph Update: In this approach, packets that are not fed back are probabilistically considered received, and their acknowledgement erased and reciprocally. This approach tends to balance stochastically between reattempting packets with unheard feedback and transmitting new packets. Since during the downlink subframe no acknowledgement is expected to be heard, and then packets are reconsidered with probability $\mathcal{P}_{B_{i}}$ and discarded with probability $\mathcal{P}_{G_{i}}$. In the uplink frame, all the uncertain packets are reviewed with probability $\tilde{\mathcal{P}}_{B_{i}}$ and removed with probability $\tilde{\mathcal{P}}_{G_{i}}$.

In the realistic graph update approach, uncertain vertices are removed from the graph with probability $\mathcal{P}_{G_{i}}$ in the downlink frame and with probability $\tilde{\mathcal{P}}_{G_{i}}$ in the uplink frame.

\section{Simulation Results}

This section shows the performance of the proposed solutions against the best-known heuristics to reduce the completion time in each scenario. In all the simulations, the different delays are computed by frame then averaged over a large number of iterations. The packet and the feedback erasure probability of all the users change from frame to frame while the average packet erasure probability remain constant. This section considers symmetric channels for both the forward and 


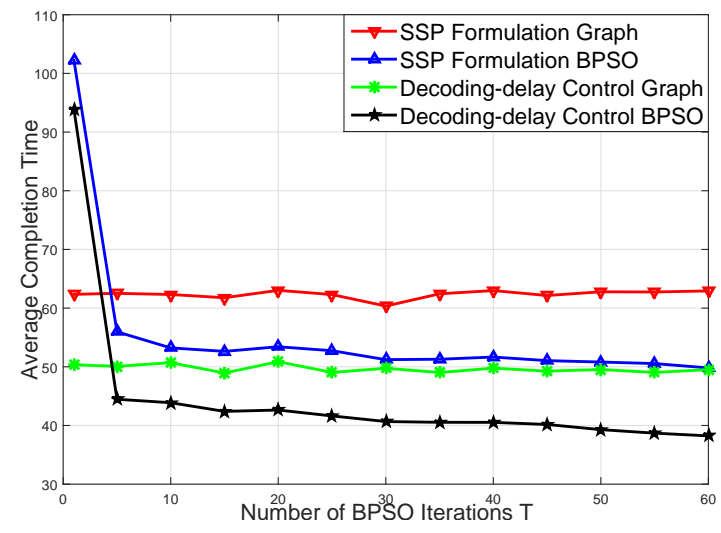

Fig. 5. Completion time versus the number of BPSO iterations $T$ for a network composed of $M=60$ users, $N=30$ packets and an average erasure probability $P=0.25$ for memoryless channels with perfect feedback.

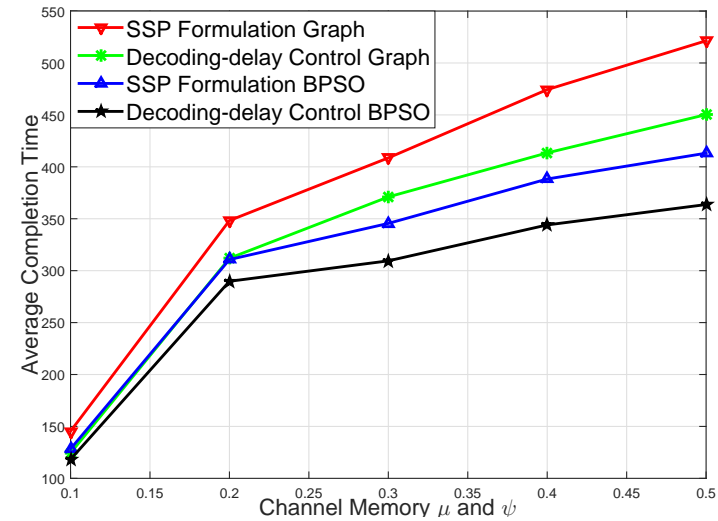

Fig. 6. Completion time versus the channel memory $\mu$ and $\psi$ for a network composed of $M=20$ users and $N=30$ packets for persistent erasure channels with imperfect feedback.

backward links. In other words, the forward and the backward links have the same parameters, e.g., $g_{i}=\tilde{g}_{i}$.

The first part of the section compares the performance of the proposed decoding-delay control approaches to the Shortest Stochastic Path approach proposed in [9] in perfect feedback scenario and memoryless channels. The memoryless channels can be obtained by setting the memory of the channel to $\mu=0$. Such channels can be characterized using an erasure probability called in this section $P$. The SSP formulation relies on solving a maximum weight clique in which the weight of a vertex $v_{i j}$ is defined as $\left|\mathcal{W}_{i}\right| /\left(1-p_{i}\right)$. To be fair in comparison, the SSP approach is tested against the proposed maximum weight clique heuristic. The BPSO approach is tested against the first heuristic.

The second part of the section compares the performance of the proposed algorithm against the blind approaches first introduced in [35] in persistent erasure channels with feedback imperfections. As shown in the previous section, such partially blind approaches rely on solving a maximum weight clique problem after discarding or reconsidering uncertain vertices. Hence, to be fair in comparison, these approaches against the proposed maximum weight heuristic. To quantify the performance of the second heuristic, this section suggests comparing the performances of both heuristics. In all simulations, following parameters are fixed: the erasure in the Good state $p=0.1$ and in the Bad stare $q=0.8$, the state switching

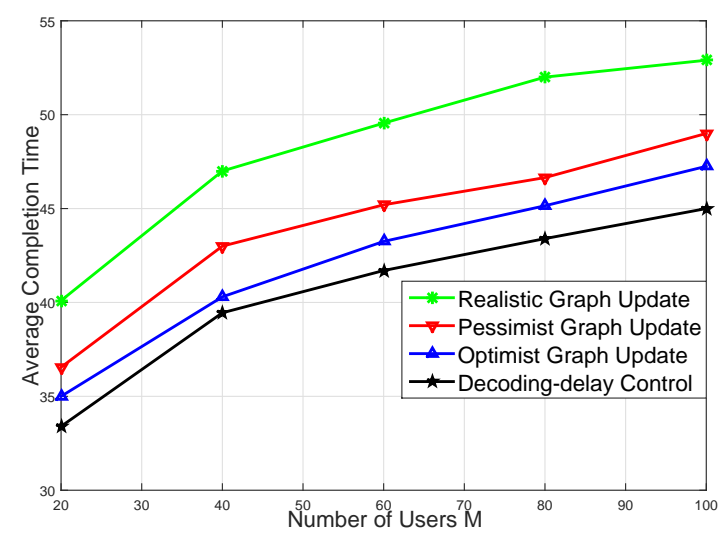

Fig. 7. Completion time versus the number of users $M$ for a network composed of $N=30$ packets and a downlink frame of duration $F=5$ for persistent erasure channels with imperfect feedback. The channel memory is $\mu=\psi=0.2$

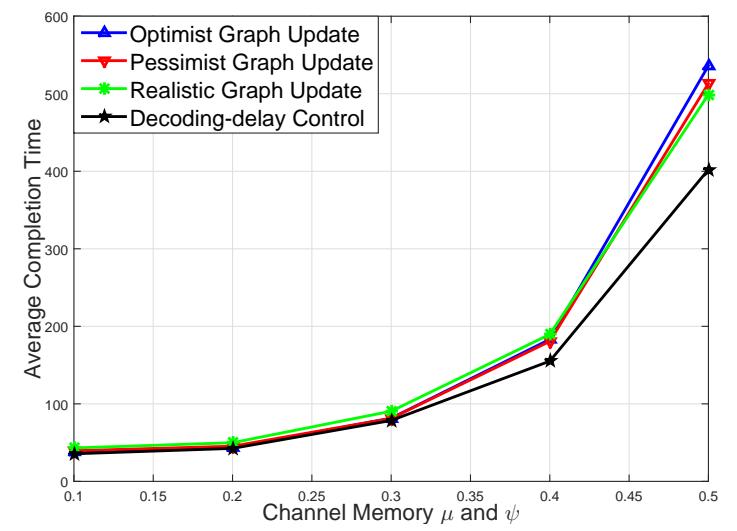

Fig. 8. Completion time versus the channel memory $\mu$ and $\psi$ for a network composed of $M=60$ users, $N=30$ packets and a downlink frame of duration $F=5$ for persistent erasure channels with imperfect feedback.

probability $b=0.2$, and the number of particles of the BPSO algorithm $L=N$. The duration of the downlink subframe is fixed to 5 time slots.

Figure 3 depicts the comparison of the mean completion for a perfect feedback and independent erasure channels against the number of users $M$ for $N=60$ and $P=0.25$. The figure shows that the proposed decoding delay approach provide appreciable gain as compared to the SSP formulation proposed in [9], [35]. Further, the gap between the two approaches becomes more importance as the number of users in the system increases. This can be explained by the light of the decoding delay control policy characteristics. In the proposed policy, the number of the erased packets is estimated using the law of large numbers. The approximation is adequate for a large system the inputs (number of packets and users).

Figure 4 depicts the same comparison for a perfect feedback and independent erasure channels against the erasure probability $P$ for $M=60$ and $N=30$. The figure clearly displays an increasing gap between the proposed approach and the SSP one. The difference between the algorithm can be explained by the fact that as the erasure of the channel increases, the decoding delay become necessary. Therefore, ignoring such effect, as the SSP approach, leads to poor performances as shown in Figure 4 for $p>0.3$.

Figure 5 illustrates the comparison of the completion time 
achieved by the maximum weight clique solution algorithm (denoted by -Graph) and the BPSO algorithm (denoted by -BPSO) for both the proposed decoding delay control and the SSP approach for a perfect feedback and independent erasure channels. The figure plots the results against against the number of iteration $T$ for $M=60, N=30$, and $P=0.25$. Again, the proposed decoding delay approach outperform the SSP one for both heuristics. Figure 6 depicts the completion time versus the channel memory $\mu$ and $\psi$ for an imperfect feedback and persistent erasure channels for a network composed of $M=20$ and $N=30$.

Both Figure 5 and Figure 6 show that the BPSO algorithm provides appreciable gain against the maximum weight clique solution for a number of iteration greater that 5 . This can be explained by the fact that the proposed maximum weight clique solution uses a simple linear algorithm to solve the problem, which may not be effective. On the other hand, BPSO intelligently explores the feasible space to find satisfactory packet combination. The gap in performance between both heuristics increases as the channel memory increases. This can be explained by the fact that the importance of satisfying the critical criterion increases along with the channel memory as the decoding delay highly depends on it. Moreover, the constant and predefined complexity property of BPSO along with its performance to reduce the completion time make this algorithm more reliable and more suitable to be used.

Figure 7 shows the performance of the proposed scheme and the different blind algorithm against the number of users $M$ for a network composed of $N=30$ packets with imperfect feedback and persistent erasure channel. Figure 8 plots the completion time for the same system inputs against the channel memory. From both figures, it can clearly be noted that the proposed decoding delay approach largely outperforms the blind approaches in all situations. The optimist approach achieves a reasonable degradation for a low channel persistence. However, this degradation becomes more severe as the memory of the channel increases. The pessimist approach can be seen as the complementary of the optimist approach since it performs better in high memory channel and less in the near independent channel. The realistic approach achieves an intermediate result and degrades as the channel is near independent or highly correlated.

\section{CONCLUSION}

This paper studies the effect of controlling the decoding delay to reduce the completion time below its currently bestknown solution for persistent channels in both perfect and imperfect feedback. The completion time reduction problem is first formulated by deriving a decoding-delay-dependent expressions of the users' and the overall completion time in the perfect feedback scenario. The paper proposes minimizing the probability of increasing the maximum of these decodingdelay-dependent completion time expressions after each transmission through a layered control of their decoding delays. The completion time reduction problem is shown to be equivalent to a maximum weight clique problem in the IDNC graph. Maximum weight clique problems being NP-hard problems, the paper proposes two moderate complexity heuristic algorithm. Finally, the decoding-delay-dependent completion time expressions are derived in imperfect feedback scenario and used to formulate the problem as a maximum weight clique problem. Simulation results show the performance of the proposed solutions and suggest that both heuristics achieves a lower mean completion time as compared to the best-known heuristics for the completion time reduction in perfect and imperfect feedback. The gap in performance becomes more significant as the erasure of the channel increases.

\section{APPENDIX A Proof of THEOREM 1}

This section approximates the completion time through a decoding delay expression. Since all derivations are relative to an arbitrary user $i$, the index $i$ is dropped in this proof. Let $\mathcal{E}(t)$ be the total number of erased transmission until time $t$. It is easy to infer that the reception completion event at time $t=C_{i}(S)$ occurs when it receives an instantly decodable packet in the $C_{i}(S)$-th recovery transmission. Consequently, $\forall t \leq \mathcal{C}_{i}(S)-1$, the transmission at time $t$ following the schedule $S$ can be one of the following options:

- The packet can be erased $\Rightarrow$ The transmission increases $\mathcal{E}(t)$, i.e., $\mathcal{E}(t)=\mathcal{E}(t-1)+1$.

- The packet can be successfully received $\Rightarrow$ Two cases can occur:

- The packet is instantly decodable. Note that the user needs to receive $|\mathcal{W}(0)|-1$ of those packets until time $t=C_{i}(S)-1$ in order to complete its reception by the last missing source packet from the transmitted packet at time $t=C_{i}(S)$. Consequently, the number of such packets until time $t=C_{i}(S)$ is equal to $|\mathcal{W}(0)|$.

- The packet is either non-innovative or non-instantly decodable $\Rightarrow$ The transmission increases the value of $D(S)$ by one.

Consequently, the number of recovery transfer following schedule $S$ until the user complete its reception of the frame packets can be expressed as follows:

$$
\mathcal{C}_{i}(S)=|\mathcal{W}(0)|+D(S)+\mathcal{E}\left(\mathcal{C}_{i}(S)-1\right) .
$$

Let $\mathcal{X}(t)$ be the number of time instant, from the beginning of the recovery phase, until the time $t$, in which the channel is in the Good state. Using the limit distribution of the Markov chain, the term can be approximated by $\mathcal{X}(t) \approx t \mathcal{P}_{G}$. Similarly, let $\mathcal{Y}(t) \approx t \mathcal{P}_{B}$ be number of time instant it is in the Bad state. Let $\mathcal{E}^{g}(t)$ and $\mathcal{E}^{b}(t)$ be the number of erased transmission in the Good and Bad state, respectively, from the beginning of the recovery phase, until the time $t$. In each state, e.g., Good and Bad, the channel acts like a memory-less binary channel. Therefore, using the law of large number in each of the states of the Markov chain yields:

$$
\begin{aligned}
& \mathcal{E}^{g}(t) \approx \mathcal{X}(t) p \approx t p \mathcal{P}_{G} \\
& \mathcal{E}^{b}(t) \approx \mathcal{Y}(t) q \approx t q \mathcal{P}_{B} .
\end{aligned}
$$

For large enough frame size $N$, the completion time $\mathcal{C}_{i}(S)$ would also be large enough and thus $\mathcal{E}\left(\mathcal{C}_{i}(S)-1\right)$ can be 
approximated by the following expression:

$$
\begin{aligned}
\mathcal{E}\left(\mathcal{C}_{i}(S)-1\right) & =\mathcal{E}^{g}\left(\mathcal{C}_{i}(S)-1\right)+\mathcal{E}^{b}\left(\mathcal{C}_{i}(S)-1\right) \\
& \approx \alpha\left(\mathcal{C}_{i}(S)-1\right),
\end{aligned}
$$

where $\alpha$ is the average erasure probability of the persistent channel defined as:

$$
\alpha=\frac{g p+q b}{g+b} .
$$

Substituting equation (A.4) in the expression of the completion time (A.1) and re-arranging the terms, the completion time for user $i$ can be finally expressed as:

$$
\mathcal{C}_{i}(S) \approx \frac{\left|\mathcal{W}_{i}(0)\right|+D_{i}(S)-\alpha_{i}}{1-\alpha_{i}}
$$

\section{APPENDIX B}

Proof OF PROPOSITION 1

This section computes the probability $e_{i}(t)$ that the transmission at time instant $t$ is erased at user $i$. Let $t_{i}^{(0)}$ be the latest time user $i$ was targeted by a transmission and let $j$ be the intended packet in the transmission. It is easy to infer that if the transmission results in a success then $f_{i j}=0$ and 1 otherwise. Let $X_{i}(t)$ is a random variable that take the value 1 if the transmission at time $t$ is erased and 0 otherwise. The probability to lose a transmission can be expressed as follows:

$$
e_{i}(t)=\left\{\begin{array}{l}
\mathbb{P}\left(X_{i}(t)=1 \mid X_{i}\left(t_{i}^{(0)}\right)=1\right) \text { if } f_{i j}=1 \\
\mathbb{P}\left(X_{i}(t)=1 \mid X_{i}\left(t_{i}^{(0)}\right)=0\right) \text { if } f_{i j}=0 .
\end{array}\right.
$$

For ease of notations, the rest of this sections computes $\mathbb{P}\left(X(n)=1 \mid X\left(n^{0}\right)=x\right), x=0,1$ and $n \geq n_{0}$. The final expression can be simply obtained by replacing developped results in (B.1).

Using the total probability theorem, the probability defined above can be expressed as follows:

$$
\begin{aligned}
& \mathbb{P}\left(X(n)=1 \mid X\left(n^{0}\right)=x\right)= \\
& \mathbb{P}\left(X(n)=1 \mid \mathcal{C}(n)=G, X\left(n^{0}\right)=x\right) \times \\
& \times \mathbb{P}\left(\mathcal{C}(n)=G \mid X\left(n^{0}\right)=x\right)+ \\
& \mathbb{P}\left(X(n)=1 \mid \mathcal{C}(n)=B, X\left(n^{0}\right)=x\right) \\
& \times \mathbb{P}\left(\mathcal{C}(n)=B \mid X\left(n^{0}\right)=x\right) .
\end{aligned}
$$

From the properties of Markov chains, the first term in (B.2), i.e., (B.2a), can be simplified as:

$$
\begin{aligned}
\mathbb{P}\left(X(n)=1 \mid \mathcal{C}(n)=G, X\left(n^{0}\right)=x\right) & = \\
& \mathbb{P}(X(n)=1 \mid \mathcal{C}(n)=G)=p .
\end{aligned}
$$

By the means of the simplification in (B.3), the probability (B.2c) can also be simplified to yield $\mathbb{P}(X(n)=1 \mid \mathcal{C}(n)=$ $\left.B, X\left(n^{0}\right)=x\right)=q$. Now develop the term (B.2b), using the total probability theorem, as follows:

$$
\begin{aligned}
\mathbb{P}\left(\mathcal{C}(n)=G \mid X\left(n^{0}\right)=x\right)= \\
\mathbb{P}\left(\mathcal{C}(n)=G \mid \mathcal{C}\left(n^{0}\right)=G, X\left(n^{0}\right)=x\right) \\
\times \mathbb{P}\left(\mathcal{C}\left(n^{0}\right)=G \mid X\left(n^{0}\right)=x\right)+ \\
\mathbb{P}\left(\mathcal{C}(n)=G \mid \mathcal{C}\left(n^{0}\right)=B, X\left(n^{0}\right)=x\right) \\
\times \\
\quad \mathbb{P}\left(\mathcal{C}\left(n^{0}\right)=B \mid X\left(n^{0}\right)=x\right) .
\end{aligned}
$$

Using a simplification similar to the one in (B.3), the previous equality (B.4) can be simplified:

$$
\begin{aligned}
& \mathbb{P}\left(\mathcal{C}(n)=G \mid X\left(n^{0}\right)=x\right)= \\
& \mathbb{P}\left(\mathcal{C}(n)=G \mid \mathcal{C}\left(n^{0}\right)=G\right) \mathbb{P}\left(\mathcal{C}\left(n^{0}\right)=G \mid X\left(n^{0}\right)=x\right)+ \\
& \mathbb{P}\left(\mathcal{C}(n)=G \mid \mathcal{C}\left(n^{0}\right)=B\right) \mathbb{P}\left(\mathcal{C}\left(n^{0}\right)=B \mid X\left(n^{0}\right)=x\right) .
\end{aligned}
$$

All the terms in (B.5) can be easily computed using the results developed in Lemma 3 and Lemma 4. Following the same steps as the one used in the simplification and computation of (B.2b), the term (B.2d) can be expressed using the results of The same steps can be used to simplified the term (B.2d) Lemma 3 and Lemma 4. Finally, rearranging the terms of the expression yields the following erasure probability:

$$
e_{i}= \begin{cases}\frac{p_{i} g_{i} \eta_{i}+q_{i} b_{i} \eta_{i}^{\prime}}{p_{i} g_{i}+q_{i} b_{i}} & \text { if } f_{i j}=1 \\ \frac{\left(1-p_{i}\right) g_{i} \eta_{i}+\left(1-q_{i}\right) b_{i} \eta_{i}^{\prime}}{\left(1-p_{i}\right) g_{i}+\left(1-q_{i}\right) b_{i}} & \text { if } f_{i j}=0 .\end{cases}
$$

where the quantities $\eta_{i}$ and $\eta_{i}^{\prime}$ are defined in (11).

\section{APPENDIX C \\ Proof of THEOREM 2}

The set $\mathcal{P}(t)$ of critical users is constructed such that it contains all users that have non-zero probabilities of increasing the completion time. Therefore, any user $j \in \mathcal{M} \backslash \mathcal{P}(t)$ is unable to increase $\max _{i \in \mathcal{M}}\left\{\mathcal{C}_{i}(t)\right\}$ compared to $\max _{i \in \mathcal{M}}\left\{\mathcal{C}_{i}(t-1)\right\}$, even if he experiences a decoding delay. According the definition of the completion time in (8), the only term that affects the completion time is the decoding delay. Hence, users $i \in \mathcal{P}(t)$ do not increase $\max _{i \in \mathcal{M}}\left\{\mathcal{C}_{i}(t)\right\}$ after the transmission $\kappa(t)$ if and only if they do not experience a decoding delay increment in that transmission. Consequently, the optimization problem (9) can be expressed as:

$$
\begin{aligned}
& \min _{\kappa \in \mathcal{G}} \mathbb{P}\left(\max _{i \in \mathcal{M}}\left\{\mathcal{C}_{i}(t)\right\}>\max _{i \in \mathcal{M}}\left\{\mathcal{C}_{i}(t-1)\right\}\right) \\
& \propto \max _{\kappa \in \mathcal{G}} \mathbb{P}\left(\max _{i \in \mathcal{M}}\left\{\mathcal{C}_{i}(t)\right\}=\max _{i \in \mathcal{M}}\left\{\mathcal{C}_{i}(t-1)\right\}\right) \\
& \propto \max _{\kappa \in \mathcal{G}} \mathbb{P}\left(\max _{i \in \mathcal{P}(t)}\left\{\mathcal{C}_{i}(t)\right\}=\max _{i \in \mathcal{M}}\left\{\mathcal{C}_{i}(t-1)\right\}\right) \\
& \propto \max _{\kappa \in \mathcal{G}} \mathbb{P}\left(d_{i}(t)=0, \forall i \in \mathcal{P}(t)\right) \\
& \propto \max _{\kappa \in \mathcal{G}} \prod_{i \in \mathcal{P}(t)} \mathbb{P}\left(d_{i}(t)=0\right) .
\end{aligned}
$$

According to the analysis done in [18], the critical criterion in (C.1) can be achieved by selecting $\kappa^{*}(t)$ according to the following optimization problem:

$$
\kappa^{*}=\arg \max _{\kappa \in \mathcal{G}}\left\{\sum_{i \in \mathcal{P}(t) \cap \tau(\kappa)} \log \left(\frac{1}{e_{i}}\right)\right\} .
$$

\section{APPENDIX D \\ PRoOF OF LEMMA 1}

This section first illustrates the poor performance of the original algorithm, i.e., a random initialization of the particles. Afterward, it provides a suitable number of particle $L$ and an appropriate initialization in order to guarantee the convergence of the overall system.

Let $L$ be the number of particles and $T$ the number of iterations. Assume there exist a user $i$ missing all the packets, 
i.e., $\mathcal{H}_{i}=\varnothing$. Further, assume that all users, expect user $i$, received all their missing packets. Therefore, the only packet combination that can reduce the Wants set of user $i$ and, therefore, update the overall system, is a packet combination in which only one packet is included. Such packet combinations are called herein a sparse packet combination as it includes a single packet. For a random initialisation, the probability that a combination is sparse is $N 2^{-N}$. Therefore, the probability that at least one combination of the $L$ particle is spare is:

$$
1-\left(1-N 2^{-N}\right)^{L}
$$

For a large number of packets $N$, with high probability none of the initial combination of the $L$ particles is sparse. For a non-sparse combination, the objective function is 0 since no user is targeted. As a consequence, the update of the position and the velocity vectors of the $L$ particles is a random update since almost all direction result in a non-sparse combination. Therefore, the second iteration of the algorithm can be seen as another initialization of the $L$ particle. The probability of at least one particle to have a spare combination after the $T$ iterations of the algorithm is:

$$
1-\left(1-N 2^{-N}\right)^{L+T}
$$

For a small number of iterations $T$, with high probability, the algorithm ends with a non-sparse combinations and, therefore, no update is made in the system. Such process results in a destitute performance of the overall system. By setting the number of particles equal to the number of packets $L=N$ and using an identity initialisation matrix, an increase in the objective function is guaranteed from the first iteration of the algorithm. Therefore, at each time instant, unless the packet is erased, the algorithm ensures a reduction by at least one packet from the Wants set of users. This concludes the overall convergence of the system independently of the number of iterations $T$ of the algorithm.

\section{APPENDIX E \\ PROOF OF THEOREM 3}

In this section, the critical criterion (9) is reformulated as a maximum weight clique problem in the IDNC graph. Following the simplification adopted in Remark 1, the time index $t$ is dropped in all of this section since it is understood that it considers finding the packet combination at the transmission $t$. Further, the innovative probability may be written without the packet argument, i.e., $p_{i, n}$, in which case it should be understood that it is the probability that the targeted packet of user $i$ to be innovative.

Using a development similar to the one used in proving Theorem 2, the critical criterion (9) can be written as follows:

$$
\max _{\kappa \in \mathcal{G}} \prod_{i \in \mathcal{P}} \mathbb{P}\left(d_{i}=0\right) \text {. }
$$

According to the analysis done in [23], the probability of the decoding delay increase for user $i$ is given by the following theorem:

Lemma 2. The probability that user $i$ does not experience a decoding delay at time $t$, after the transmission $\kappa$ is:

$$
\begin{aligned}
& \mathbb{P}\left(d_{i}=0\right) \\
& = \begin{cases}e_{i} & i \in(\widehat{\tau} \cap \bar{F}) \\
e_{i}+p_{i, f}-e_{i} p_{i, f} & i \in(\widehat{\tau} \cap F) \\
1 & i \in(\tau \cap \bar{U}) \\
e_{i}+p_{i, n}-e_{i} p_{i, n} & i \in(\tau \cap(U \backslash F)) \\
e_{i}+\left(1-e_{i}\right)\left(p_{i, n}+p_{i, f}\right) & i \in(\tau \cap F) .\end{cases}
\end{aligned}
$$

where $\widehat{\tau}=M_{w} \backslash \tau$ is set of users not targeted and having non-empty Wants sets, $F$ is the set of users having all their remaining packets in an uncertain state and $U$ is the set of users having the intended packet for them in an uncertain state. The notation $\bar{X}$ refers to the set complementary to the set $X$.

Proof: The proof is omitted as it mirrors the steps used in proving Theorem 2 in [23].

From the expression of the decoding delay increase in Lemma 2 and since the function $\log ($.$) is an increasing func-$ tion, the completion time reduction problem can be formulated as follows:

$$
\begin{aligned}
\kappa^{*} & =\arg \max _{\kappa \in \mathcal{G}}\left\{\sum_{i \in(\mathcal{P} \cap \widehat{\tau} \cap \bar{F})} \log \left(e_{i}\right)\right. \\
& +\sum_{i \in(\mathcal{P} \cap \widehat{\tau} \cap F)} \log \left(e_{i}+p_{i, f}-e_{i} p_{i, f}\right) \\
& +\sum_{i \in(\mathcal{P} \cap \tau \cap(U \backslash F))} \log \left(e_{i}+p_{i, n}-e_{i} p_{i, n}\right) \\
& \left.+\sum_{i \in(\mathcal{P} \cap \tau \cap F)} \log \left(e_{i}+\left(1-e_{i}\right)\left(p_{i, n}+p_{i, f}\right)\right)\right\} .
\end{aligned}
$$

In the rest of the section, the expression proposed in (E.3) is simplified so as to reformulated the problem as a maximum weight clique problem in the IDNC graph. First note that if user $i$ does not have all its wanted packets in an uncertain state, then the probability that he finished receiving all its desired packet is 0 . Thus $p_{i, f}=0, \forall i \in \bar{F}$. Therefore, the subequation (E.3a) can be expressed as follows:

$$
\sum_{i \in(\mathcal{P} \cap \widehat{\tau} \cap \bar{F})} \log \left(e_{i}\right)=\sum_{i \in(\mathcal{P} \cap \widehat{\tau} \cap \bar{F})} \log \left(e_{i}+p_{i, f}-e_{i} p_{i, f}\right) .
$$

Using the simplification proposed in (E.4), sub-equations (E.3a) and (E.3b) may be unified as follows:

$$
\begin{aligned}
& \sum_{i \in(\mathcal{P} \cap \widehat{\tau} \cap \bar{F})} \log \left(e_{i}\right)+\sum_{i \in(\mathcal{P} \cap \widehat{\tau} \cap F)} \log \left(e_{i}+p_{i, f}-e_{i} p_{i, f}\right) \\
= & \sum_{i \in(\mathcal{P} \cap \widehat{\tau})} \log \left(e_{i}+p_{i, f}-e_{i} p_{i, f}\right) .
\end{aligned}
$$

It is clear that $(U \backslash F) \subseteq \bar{F}$, then $p_{i, f}=0, \forall i \in(U \backslash F)$. Therefore, sub-equation (E.3c) can be written as follows:

$$
\begin{aligned}
& \sum_{i \in(\mathcal{P} \cap \tau \cap(U \backslash F))} \log \left(e_{i}+p_{i, n}-e_{i} p_{i, n}\right) \\
& =\sum_{i \in(\mathcal{P} \cap \tau \cap(U \backslash F))} \log \left(e_{i}+\left(1-e_{i}\right)\left(p_{i, n}+p_{i, f}\right)\right) .
\end{aligned}
$$

Using the development in (E.6), both sub-equations (E.3c) and 
(E.3d) can be written with a single equation as follows:

$$
\begin{aligned}
& \quad \sum_{i \in(\mathcal{P} \cap \tau \cap(U \backslash F))} \log \left(e_{i}+p_{i, n}-e_{i} p_{i, n}\right) \\
& \quad+\sum_{i \in(\mathcal{P} \cap \tau \cap F)} \log \left(e_{i}+\left(1-e_{i}\right)\left(p_{i, n}+p_{i, f}\right)\right) \\
& =\sum_{i \in(\mathcal{P} \cap \tau \cap U)} \log \left(e_{i}+\left(1-e_{i}\right)\left(p_{i, n}+p_{i, f}\right)\right) .
\end{aligned}
$$

Given the above simplifications in (E.5) and (E.7), the completion time minimization problem can be expressed as follows:

$$
\begin{aligned}
\kappa^{*} & =\arg \max _{\kappa \in \mathcal{G}}\left\{\sum_{i \in(\mathcal{P} \cap \widehat{\tau})} \log \left(e_{i}+p_{i, f}-e_{i} p_{i, f}\right)\right. \\
& \left.+\sum_{i \in(\mathcal{P} \cap \tau \cap U)} \log \left(e_{i}+\left(1-e_{i}\right)\left(p_{i, n}+p_{i, f}\right)\right)\right\} \\
& =\arg \min _{\kappa \in \mathcal{G}}\left\{\sum_{i \in(\mathcal{P} \cap \tau)} \log \left(e_{i}+p_{i, f}-e_{i} p_{i, f}\right)\right. \\
& \left.-\sum_{i \in(\mathcal{P} \cap \tau \cap U)} \log \left(e_{i}+\left(1-e_{i}\right)\left(p_{i, n}+p_{i, f}\right)\right)\right\} .
\end{aligned}
$$

Note that if the targeted packet $\kappa_{i}$ of user $i$ is not an uncertain packet, i.e., $i \in \bar{U}$, then the packet is certainly innovative. Since that this user have at least one certain wanted packet then he surely still needs packets. In other words, we have $i \in \bar{U} \Rightarrow p_{i, n}=1$ and $p_{i, f}=0$. We write the following expression as:

$$
\begin{aligned}
& \sum_{i \in(\mathcal{P} \cap \tau)} \log \left(e_{i}+\left(1-e_{i}\right)\left(p_{i, n}+p_{i, f}\right)\right) \\
= & \sum_{i \in(\mathcal{P} \cap \tau \cap U)} \log \left(e_{i}+\left(1-e_{i}\right)\left(p_{i, n}+p_{i, f}\right)\right) .
\end{aligned}
$$

Finally, substituting (E.9) in (E.8), the completion time reduction problem in persistent erasure channel with imperfect feedback can be written as:

$$
\begin{aligned}
\kappa^{*} & =\arg \min _{\kappa \in \mathcal{G}}\left\{\sum_{i \in(\mathcal{P} \cap \tau)} \log \left(\frac{e_{i}+p_{i, f}-e_{i} p_{i, f}}{e_{i}+\left(1-e_{i}\right)\left(p_{i, n}+p_{i, f}\right)}\right)\right\} . \\
& =\arg \max _{\kappa \in \mathcal{G}}\left\{\sum_{i \in(\mathcal{P} \cap \tau)} \log \left(\frac{e_{i}+\left(1-e_{i}\right)\left(p_{i, n}+p_{i, f}\right)}{e_{i}+p_{i, f}-e_{i} p_{i, f}}\right)\right\} \\
& =\arg \max _{\kappa \in \mathcal{G}}\left\{\sum_{i \in(\mathcal{P} \cap \tau)} \log \left(1+\frac{p_{i, n}}{\frac{e_{i}}{1-e_{i}}+p_{i, f}}\right)\right\} .
\end{aligned}
$$

In other words, the transmission $\kappa$ than can satisfy the critical criterion can be selected using a maximum weight clique problem in which the weight of each vertex $v_{i j}$ are given in (20).

\section{APPENDIX F \\ AUXILIARY LEMMAS}

This section provides auxiliary lemmas computing the probability of being in one of the states of the persistent channels under investigation, illustrated in Figure 2. The results developed in this section are used in the proof of Proposition 1. For notation convenience, the index of the user is dropped in this section as it focuses on a single one. Let $X(n)$ be a random variable that takes the value 1 if the transmission at time $n$ is erased and 0 otherwise. The first lemma computes the probability to be in a particular state given the channel was in a given state in a previous transmission. The second lemma computes the conditional probability of being in a given state conditioned by the channel realization.

Lemma 3. The probability of the channel to be in the Good (Bad) state at transmission $n$ given it was in the Bad (Good) state at transmission $n^{(0)}<n$ is:

$$
\begin{gathered}
\mathbb{P}\left(\mathcal{C}(n)=G \mid \mathcal{C}\left(n^{(0)}\right)=B\right)=g \sum_{i=0}^{n-n^{(0)}-1} \mu^{i} \\
\mathbb{P}\left(\mathcal{C}(n)=B \mid \mathcal{C}\left(n^{(0)}\right)=G\right)=b \sum_{i=0}^{n-n^{(0)}-1} \mu^{i} .
\end{gathered}
$$

Proof: The proof of this lemma is omitted as it mirrors the steps used in proving Appendix A of [23].

Lemma 4. The probability of the channel to be in the Good state conditioned by the realization $X(n)$ can be expressed as:

$$
\begin{aligned}
& \mathbb{P}(\mathcal{C}(n)=G \mid X(n)=1)=\frac{p g}{p g+q b} \\
& \mathbb{P}(\mathcal{C}(n)=G \mid X(n)=0)=\frac{(1-p) g}{(1-p) g+(1-q) b}
\end{aligned}
$$

Proof: To prove this lemma, let first apply the total probability theorem to show $\mathbb{P}\left(X_{i}(n)=x\right), x=1,0$ as follows:

$$
\mathbb{P}\left(X_{i}(n)=x\right)= \begin{cases}p \mathcal{P}_{G}+q \mathcal{P}_{B} & \text { if } x=1 \\ (1-p) \mathcal{P}_{G}+(1-q) \mathcal{P}_{B} & \text { if } x=0 .\end{cases}
$$

The erasure probability conditioned by the channel state is, by definition of the persistent channel, the following:

$$
\mathbb{P}\left(X_{i}(n)=x \mid \mathcal{C}(n)=G\right)= \begin{cases}p & \text { if } x=1 \\ (1-p) & \text { if } x=0 .\end{cases}
$$

Reformulating the conditional probability using Bayes theorem yields:

$$
\mathbb{P}\left(\mathcal{C}(n)=G \mid X_{i}(n)=x\right)=\frac{\mathcal{P}_{G} \mathbb{P}\left(X_{i}(n)=x \mid \mathcal{C}(n)=G\right)}{\mathbb{P}\left(X_{i}(n)=x\right)} .
$$

The final result can be just obtained by a simple substitution of (F.5) and (F.6) in the previous expression and replacing $\mathcal{P}_{G}$ and $\mathcal{P}_{B}$ by their expressions defined in (3).

\section{REFERENCES}

[1] A. Douik, S. Sorour, M.-S. Alouini, and T. Y. Al-Naffouri, "Completion time reduction in instantly decodable network coding through decoding delay control," in Proc. of IEEE Global Telecommunications Conference (GLOBECOM' 2014), Austin, Texas, USA, Dec. 2014.

[2] R. Ahlswede, N. Cai, S.-Y. Li, and R. Yeung, "Network information flow," IEEE Transactions on Information Theory, vol. 46, no. 4, pp. 1204-1216, Jul 2000 
[3] A. Tassi, I. Chatzigeorgiou, and D. Vukobratovic, "Resource-allocation frameworks for network-coded layered multimedia multicast services," IEEE Journal on Selected Areas in Communications, vol. 33, no. 2, pp. 141-155, Feb 2015

[4] M. S. Karim, P. Sadeghi, S. Sorour, and N. Aboutorab, "Instantly decodable network coding for real-time scalable video broadcast over wireless networks," EURASIP Journal on Advances in Signal Processing, vol. 2016, no. 1, pp. 1-24, 2016.

[5] L. Lu, M. Xiao, and L. K. Rasmussen, "Design and analysis of relayaided broadcast using binary network codes," Journal of Communications, vol. 6, no. 8, pp. 610-617, 2011.

[6] X. Li, C.-C. Wang, and X. Lin, "Optimal immediately-decodable intersession network coding (IDNC) schemes for two unicast sessions with hard deadline constraints," in Proc of 49th Annual Allerton Conference on Communication, Control, and Computing (Allerton' 2011), Monticello, IL, USA, Sept 2011, pp. 784-791.

[7] E. Drinea, C. Fragouli, and L. Keller, "Delay with network coding and feedback," in Proc. of IEEE International Symposium on Information Theory (ISIT' 2009), Seoul, Korea, June 2009, pp. 844-848.

[8] A. Douik, S. Sorour, M.-S. Alouini, and T. Al-Naffouri, "Delay reduction in lossy intermittent feedback for generalized instantly decodable network coding," in Proc. of IEEE 9th International Conference on Wireless and Mobile Computing, Networking and Communications (WiMob' 2013), Lyon, France, Oct 2013, pp. 388-393.

[9] S. Sorour and S. Valaee, "Completion delay minimization for instantly decodable network codes," IEEE/ACM Transactions on Networking, vol. 23 , no. 5 , pp. $1553-1567$, Oct 2015 .

[10] — "Minimum broadcast decoding delay for generalized instantly decodable network coding," in Proc. of IEEE Global Telecommunications Conference (GLOBECOM’2010), Miami, Florida, USA, Dec 2010, pp. $1-5$.

[11] A. Douik, S. Sorour, T. Y. Al-Naffouri, and M.-S. Alouini, "Instantly decodable network coding for real-time device-to-device communications," EURASIP Journal on Advances in Signal Processing, vol. 2016, no. 1, pp. 1-14, 2016.

[12] A. Douik, S. Sorour, T. Y. Al-Naffouri, H.-C. Yang, and M.-S. Alouini, "Delay reduction in Multi-Hop Device-to-Device communication using network coding," in Proc. of IEEE International Symposium on Network Coding (NetCod' 2015), Sydney, Australia.

[13] A. Douik, S. Sorour, T. Y. Al-Naffouri, and M.-S. Alouini, "Delay reduction in Multi-Hop Device-to-Device communication using network coding," submitted to IEEE Transactions on Communication, available Arxiv e-print, vol. abs/1409.8460, 2014.

[14] A. Douik, S. Sorour, H. Tembine, T. Y. Al-Naffouri, and M.-S. Alouini, "A game-theoretic framework for decentralized cooperative data exchange using network coding," accepted in IEEE Transaction on Mobile Computing, available Arxiv e-print, vol. abs/1404.3637, 2014.

[15] H. Khamfroush, D. E. Lucani, P. Pahlevani, and J. Barros, "On optimal policies for network-coded cooperation: Theory and implementation," IEEE Journal on Selected Areas in Communications, vol. 33, no. 2, pp. 199-212, Feb 2015.

[16] A. Douik, S. Sorour, H. Tembine, T. Y. Al-Naffouri, and M.-S. Alouini, "A game theoretic approach to minimize the completion time of network coded cooperative data exchange," in Proc. of IEEE Global Telecommunications Conference (GLOBECOM' 2014), Austin, Texas, USA, Dec. 2014.

[17] N. Aboutorab, P. Sadeghi, and S. Sorour, "Enabling a tradeoff between completion time and decoding delay in instantly decodable network coded systems," IEEE Transactions on Communications, vol. 62, no. 4, pp. 1296-1309, April 2014.

[18] A. Douik, S. Sorour, M. Alouini, and T. Y. Al-Naffouri, "On minimizing the maximum broadcast decoding delay for instantly decodable network coding," Proc. of IEEE Vehicular Technology Conference (VTC-Fall' 2014), Vancouver, BC, Canada.

[19] P. Sadeghi, D. Traskov, and R. Koetter, "Adaptive network coding for broadcast channels," in Proc. of Workshop on Network Coding, Theory, and Applications (NetCod' 2009), Lausanne, Switzerland, June 2009, pp. $80-85$.

[20] S. Sorour and S. Valaee, "Completion delay reduction in lossy feedback scenarios for instantly decodable network coding," in Proc. of IEEE 22nd International Symposium on Personal Indoor and Mobile Radio Communications (PIMRC' 2011), Toronto, Canada, Sept 2011, pp. 2025-2029.

[21] S. Sorour, A. Douik, S. Valaee, T. Al-Naffouri, and M. Alouini, "Partially blind instantly decodable network codes for lossy feedback environment," IEEE Transactions on Wireless Communications, vol. 13, no. 9, pp. 4871-4883, Sept 2014.
[22] M. Esmaeilzadeh and P. Sadeghi, "Optimizing completion delay in network coded systems over TDD erasure channels with memory," in Proc. of IEEE International Symposium on Communications and Information Technologies (ISCIT' 2012), Queensland, Australia, Oct 2012, pp. 883-888.

[23] A. Douik, S. Sorour, T. Y. Al-Naffouri, and M. S. Alouini, "Delay reduction for instantly decodable network coding in persistent channels with feedback imperfections," IEEE Transactions on Wireless Communications, vol. 14, no. 11, pp. 5956-5970, Nov 2015.

[24] A. Le, A. Tehrani, A. Dimakis, and A. Markopoulou, "Instantly decodable network codes for real-time applications," in Proc of International Symposium on Network Coding (NetCod' 2013), Calgary, Canada, June 2013, pp. 1-6.

[25] P. Sadeghi, R. Shams, and D. Traskov, "An optimal adaptive network coding scheme for minimizing decoding delay in broadcast erasure channels," EURASIP Journal on Wireless Communications and Networking, vol. 2010, pp. 1-14, 2010.

[26] E. O. Elliott, "Estimates of error rates for codes on burst-noise channels," Bell System Technical Journal, vol. 42, no. 5, pp. 1977-1997, 1963.

[27] M. Mushkin and I. Bar-David, "Capacity and coding for the gilbertelliott channels," IEEE Transactions on Information Theory, vol. 35 , no. 6, pp. 1277-1290, Nov 1989.

[28] P. Sadeghi, R. Kennedy, P. Rapajic, and R. Shams, "Finite-state markov modeling of fading channels - a survey of principles and applications," IEEE Signal Processing Magazine, vol. 25, no. 5, pp. 57-80, September 2008.

[29] P. R. J. Ostergard, "A fast algorithm for the maximum clique problem," Discrete Appl. Math, vol. 120, pp. 197-207.

[30] K. Yamaguchi and S. Masuda, "A new exact algorithm for the maximumweight clique problem," in Proc. Of the 23rd International Technical Conference on Circuits/Systems, Computers and Communications (ITCCSCC' 2008), Yamaguchi, Japan.

[31] Z. Akbari, "A polynomial-time algorithm for the maximum clique problem," in Proc. of IEEE/ACIS 12th International Conference on Computer and Information Science (ICIS' 2013), Niigata, Japan, June 2013, pp. 503-507.

[32] J. Kennedy and R. Eberhart, Swarm Intelligence. Morgan Kaufmann Publishers, Inc., 2001.

[33] M. Khanesar, M. Teshnehlab, and M. Shoorehdeli, "A novel binary particle swarm optimization," in Proc. of the Mediterranean Conference on Control Automation (MED' 2007), Pisa, Italy, June 2007, pp. 1-6.

[34] A. Douik, S. Sorour, T. Al-Naffouri, and M.-S. Alouini, "A lossy graph model for delay reduction in generalized instantly decodable network coding," IEEE Wireless Communications Letters, vol. 3, no. 3, pp. 281284, June 2014.

[35] S. Sorour and S. Valaee, "On minimizing broadcast completion delay for instantly decodable network coding," in Proc. of IEEE International Conference on Communications (ICC' 2010), Cape Town, South Africa, May 2010, pp. 1-5.



Ahmed Douik (S'13) received the Eng. degree in electronic and communication engineering (with first class honors) from the Ecole Polytechnique de Tunisie, Tunisia, in 2013, the M.S. degree in electrical engineering from King Abdullah University of Science and Technology, Thuwal, Saudi Arabia, in 2015. He is now pursuing his Ph.D. at the California Institute of Technology, Pasadena, CA, USA. His research interests include cloud-radio access networks, network coding, single and multi-hop transmissions, and cooperation communication. 


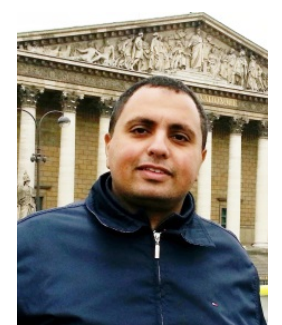

Sameh Sorour (S98-M11-SM16) is an Assistant Professor at the Department of Electrical and Computer Engineering, University of Idaho. He received his B.Sc. and M.Sc. degrees in Electrical Engineering from Alexandria University, Egypt, in 2002 and 2006, respectively. In 2011, he obtained his $\mathrm{Ph} . \mathrm{D}$. degree in Electrical and Computer Engineering from University of Toronto, Canada. After two postdoctoral fellowships at University of Toronto and King Abduallah University of Science and Technology (KAUST), he joined King Fahd University of Petroleum and Minerals (KFUPM) in 2013 before moving to University of Idaho in 2016. His research interests include network coding, data dissemination and exchange for the Internet of Things, cloud storage networks, femtocaching, smart management of networks and cities, and indoor positioning.

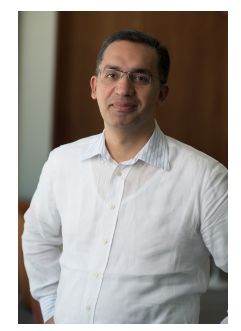

Tareq Y. Al-Naffouri (M'10) Tareq Al-Naffouri received the B.S. degrees in mathematics and electrical engineering (with first honors) from King Fahd University of Petroleum and Minerals, Dhahran, Saudi Arabia, the M.S. degree in electrical engineering from the Georgia Institute of Technology, Atlanta, in 1998, and the Ph.D. degree in electrical engineering from Stanford University, Stanford, CA, in 2004.

He was a visiting scholar at California Institute of Technology, Pasadena, CA, from January to August 2005 and during summer 2006. He was a Fulbright scholar at the University of Southern California from February to September 2008. He has held internship positions at NEC Research Labs, Tokyo, Japan, in 1998, Adaptive Systems Lab, University of California at Los Angeles in 1999, National Semiconductor, Santa Clara, CA, in 2001 and 2002, and Beceem Communications Santa Clara, CA, in 2004. He is currently an Associate at the Electrical Engineering Department, King Abdullah University of Science and Technology (KAUST). His research interests lie in the areas of sparse, adaptive, and statistical signal processing and their applications and in network information theory. He has over 150 publications in journal and conference proceedings, 9 standard contributions, 10 issued patents, and 6 pending.

Dr. Al-Naffouri is the recipient of the IEEE Education Society Chapter Achievement Award in 2008 and Al-Marai Award for innovative research in communication in 2009. Dr. Al-Naffouri has also been serving as an Associate Editor of Transactions on Signal Processing since August 2013.

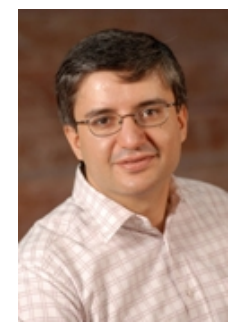

Mohamed-Slim Alouini (S'94-M'98-SM'03-F'09) was born in Tunis, Tunisia. He received the Ph.D. degree in electrical engineering from the California Institute of Technology (Caltech), Pasadena, CA, USA, in 1998. He served as a faculty member at the University of Minnesota, Minneapolis, MN, USA, then in the Texas A\&M University at Qatar, Education City, Doha, Qatar, before joining King Abdullah University of Science and Technology (KAUST), Thuwal, Makkah Province, Saudi Arabia, as a Professor of electrical engineering in 2009. His current research interests include the modeling, design, and performance analysis of wireless communication systems. 